Artigo Original

\title{
O Índice de Desenvolvimento Humano e a Data de Nascimento podem condicionar a ascensão de jogadores de Futebol ao alto nível de rendimento?
}

\author{
Israel Teoldo da Costa ${ }^{1}$ \\ Felippe da Silva Leite Cardoso ${ }^{1}$ \\ Júlio Garganta ${ }^{2}$ \\ ${ }^{1}$ Núcleo de Pesquisa e Estudos em Futebol, Universidade Federal de Viçosa, Viçosa, MG, Brasil \\ ${ }^{2}$ Centro de Investigação, Formação, Inovação e Intervenção em Desporto, CIFI $D$, Porto, Portugal
}

Resumo: $O$ presente trabalho objetiva identificar a possível influência do Índice de Desenvolvimento Humano (IDH) e da data de nascimento (DN) na ascensão de jogadores ao alto nível de rendimento. A amostra integra os 643 jogadores que disputaram o Campeonato Brasileiro Série A de 2010. Foram coletados dados referentes à data e ao local de nascimento juntamente com seu IDH. Foi utilizada a estatística descritiva e os testes Kolmogorov-Smirnov, Qui-quadrado, Kruskall-Wallis e coeficiente de corelação de Pearson. O nível de significância adotado foi de $\alpha=0,05$. Os resultados sugerem que jogadores nascidos em cidades com valores populacionais inferiores a 100 mil habitantes, com IDH médio (entre $0,501-0,800)$ e, nascidos nos primeiros meses do ano, apresentam maiores chances de atingir a elite. Afigura-se plausível concluir que a DN e o IDH são fatores que associados podem determinar a ascensão de jogadores ao alto rendimento.

Palavras-chave: Futebol. Índice de Desenvolvimento Humano. Idade Relativa. Taxa Demográfica.

\section{Could the Human Development Index and Birth-Date of the players influence the climbing up to higher performance in Soccer?}

\begin{abstract}
The present study verifies the possible influence of the human development index (HDI) and the birth-date (BD) on the climb up to higher performance in sports, considering Brazilian football context. The sample comprised all 643 players from 20 teams of the Premier Football League in Brazil. The data were collected from official website of Brazilian National Federation, United Nations Development Programme and Brazilian Institute of Geography and Statistics. Descriptive analysis, Kolmogorov-Smirnov test, chi-square test $\left(\chi^{2}\right)$, Pearson correlation and Kruskall-Wallis $\mathrm{H}$ test were carried out. The level of significance was set at $\alpha=0.05$. The results suggested that contextual factors associated with size and HDI of birth-place and birthdate contribute to the achievement of the Premier Football League. Thus, players were born in the first months of the year and in cities with medium or higher HDI had more conditions to climb up the elite level of Brazilian Football.
\end{abstract}

Keywords: Soccer. Human Development Index. Relative Age. Demographic Rate.

\section{Introdução}

O rendimento esportivo é condicionado pela expressão de capacidades táticas, técnicas, físicas, cognitivas, biotipológicas e por constrangimentos socioambientais. Neste contexto, as diferenças individuais $\mathrm{e}$ as oportunidades e experiências ambientais vivenciadas durante a infância podem conduzir a disparidades no processo de formação esportiva (BLOOM, 1985; CÔTÉ et al., 2006).

Entre as oportunidades e experiências ambientais, os aspectos sócio-econômicos referentes às taxas demográficas e ao Índice de Desenvolvimento Humano (IDH), parecem condicionar o tipo de atividades vivenciadas, a motivação intrínseca e extrínseca para prática esportiva e a qualidade de desenvolvimento das habilidades essenciais para 0 rendimento esportivo. Num estudo realizado por Côté e colaboradores (2006), foi verificado que crianças nascidas em cidades maiores têm mais acesso a recursos quando comparados com os seus homólogos de cidades menores. Os autores pontuaram que atletas oriundos de centros urbanos maiores dispõem de mais possibilidades para praticar o esporte em um ambiente estruturado, tal como uma liga, onde geralmente se possui a orientação qualificada de treinadores com horas de prática e jogos específicos, do que indivíduos advindos de cidades menores, onde as estruturas e o nível competitivo das competições 
podem não atingir uma qualidade tão elevada. Além disto, evidências científicas têm sugerido que a formação de atletas de elite geralmente se inicia em ambientes propícios à experimentação regular de atividades esportivas nos primeiros anos de vida (BAKER; CÔTÉ; ABERNETHY, 2003; SOBERLAK; CÔTÉ, 2003). Assim, o acesso à prática esportiva qualificada e bem orientada nesta etapa da formação esportiva pode ser decisivo para o sucesso esportivo de crianças e jovens.

No que refere às diferenças individuais, os efeitos da idade relativa (EIR's) vêm sendo estudados desde a década de 1970 (WILLIAMS et al., 1970; BARNSLEY; THOMPSON; BARNSLEY, 1985; MALINA, 1994; HELSEN; STARKES; VAN WINCKEL, 2000; MUJIKA et al., 2009). Trata-se de um conceito que se reporta às diferenças de idade cronológica entre os indivíduos que estão agrupados dentro de uma determinada faixa etária, podendo resultar em (des)vantagens de oportunidade para a prática que podem conduzir a diferenças significativas no desempenho esportivo (BARNSLEY; THOMPSON; BARNSLEY, 1985; $\underline{\text { COBLEY; }}$ ABRAHAM; BAKER, 2008).

O agrupamento por idade é um método recorrentemente utilizado no esporte e também em escolas, na tentativa de atenuar as diferenças físicas e cognitivas entre os jogadores e alunos que competem em determinada categoria (MUSCH; GRONDIN, 2001). Entretanto, tal estratégia não parece estar sendo suficientemente eficaz para prevenir os EIR's, de forma a promover uma competição mais justa e a proporcionar equidade de condições para todos conseguirem atingir o sucesso (HELSEN; VAN WINCKEL; WILLIAMS, 2005). Investigações realizadas no contexto do Futebol, que dividiram as datas de nascimento da população em quatro trimestres, mostraram que os jogadores que nasceram no início do ano são mais susceptíveis de serem selecionados para jogar em uma equipe, devido às suas vantagens físicas e pelo fato de serem mais experientes do que aqueles jogadores que nasceram no final do ano (MUSCH; HAY, 1999; HELSEN; STARKES; VAN WINCKEL, 2000). Segundo alguns pesquisadores, os EIR's podem levar a uma maior percepção de competência, auto-eficácia, motivação e a outros aspectos cognitivos que, por sua vez, têm um impacto sobre a qualidade da aprendizagem e sobre o nível de desempenho dos jogadores (WILLIAMS; ERICSSON, 2005; CÔTÉ et al., 2006; ASHWORTH; HEYNDELS, 2007). Como resultado, as pesquisas têm sugerido que as crianças nascidas logo no início do ano, em média, possuem um desempenho melhor do que seus pares que nasceram no final do ano e este fator tem aumentado a probabilidade destas crianças se envolverem no esporte e chegarem ao alto nível de rendimento (SHEARER, 1967; HELSEN; VAN WINCKEL; WILLIAMS, 2005). Desta forma, as vantagens físicas e cognitivas que decorrem da data de nascimento dos jogadores podem se tornar fatores determinantes para a manifestação dos EIR's nas categorias de base do futebol, perdurando até à fase adulta (VAEYENS; PHILIPPAERTS; MALINA, 2005; MUJIKA et al., 2009).

Além disso, as datas de nascimento podem aumentar a probabilidade dos jogadores nascidos no princípio do ano serem identificados como talentosos e, portanto, apresentarem maior tendência para serem transferidos para clubes da primeira divisão, o que irá propiciar treinamentos de alta qualidade e oportunidades de competição de alto nível (MALINA; BOUCHARD; BAR-OR, 2004; JOHNSON; DOHERTY; FREEMONT, 2009). Adicionalmente, ao considerar que estes clubes possuem melhores infra-estruturas, maiores intensidades de treinamento e elevados níveis de competição, o resultado será refletido em uma maior produtividade dos jogadores (ASHWORTH; HEYNDELS, 2007). Nesta perspectiva, é possível verificar que a identificação e o recrutamento de jogadores talentosos irão resultar numa educação desportiva que irá beneficiar o desenvolvimento das habilidades esportivas do jogador de Futebol (REILLY et al., 2000).

Diante do exposto, o presente trabalho tem por objetivo identificar a possível influência do Índice de Desenvolvimento Humano (IDH) e da data de nascimento (DN) na ascensão de jogadores ao alto nível de rendimento, no contexto do futebol brasileiro.

\section{Metodologia}

\section{Amostra}

A amostra integra todos os 643 de jogadores pertencentes aos 20 clubes que disputaram 0 Campeonato Brasileiro Série A de 2010. Estes jogadores possuíam média de idade igual a $25,59 \pm 4,64$ anos e eram provenientes de cidades 
com uma população média de 1.984.633,73 $\pm 3.407 .714,74$ habitantes e com um IDH médio de $0,73 \pm 0,08$. Em relação aos estatutos posicionais dos jogadores observa-se que a amostra possui 73 goleiros, 209 defensores, 234 meio-campistas e 127 atacantes.

\section{Procedimento de Recolha de dados}

Os dados referentes ao local e data de nascimento dos jogadores foram coletados nos sites oficiais dos 20 clubes participantes da competição e da Confederação Brasileira de Futebol (CBF) (http://www.cbf.com.br). Eles foram comparados no momento de aferição da fidedignidade no pareamento entre estas duas fontes. No caso de se constar divergência de informação, os dados seriam excluídos da amostra.

No que concerne ao IDH e a população das cidades, os dados foram coletados, respectivamente, nos sites oficiais do Programa das Nações Unidas para o Desenvolvimento (PNUD) (http://www.pnud.org.br) e do Instituto Brasileiro de Geografia e Estatística (IBGE) (http://www.ibge.gov.br). Para avaliação destas variáveis foram considerados os dados dos censos de 1990, devido ao fato da primeira publicação do IDH pelo PNUD ter sido realizada neste ano e também por estes dados representarem maior proximidade com a data de nascimento da maioria dos jogadores que estavam inscritos no Campeonato Brasileiro de 2010 Série $A$, quando comparados com as outras publicações de IDH feitas pelo PNUD.

\section{Análise Estatística}

Foram realizadas análises descritivas (frequência, percentual, média, desvio padrão) para se obter informações sobre diferentes aspectos da amostra. O teste de KolmogorovSmirnov foi utilizado para verificar a normalidade de distribuição dos dados. Os dados do IDH foram divididos em três intervalos $[T 1(<0,500)$, T2 $(0,501-0,800)$ e T3(>0,801)], conforme sugestões apresentadas por Asher e Daponte (2010); e os dados da DN foram divididos em trimestres [ $1^{\circ} \mathrm{T}$ (Jan-Mar), $2^{\circ} \mathrm{T}$ (Abr-Jun), $3^{\circ} \mathrm{T}$ (JulSet) e $4 \div$ T(Out-Dez)], seguindo o intervalo de datas de $1^{\circ}$ de Janeiro à 31 de Dezembro aplicado pela Fédération Internationale de Football Association (FIFA) para todas as competições internacionais. O teste do Quiquadrado foi utilizado para verificar diferenças entre as distribuições das DN's pelos trimestres e dos IDH's pelos três intervalos. Verificando que a distribuição dos dados não foi homogênea, utilizou-se $\mathrm{O}$ teste de Kruskall-Wallis $\mathrm{H}$ para comparar os valores de IDH obtidos pelas vinte equipes participantes do Campeonato Brasileiro e - coeficiente de correlação de Pearson foi utilizada para verificar as associações entre o IDH, a concentração populacional das cidades e a DN dos jogadores. Todos os procedimentos estatísticos utilizaram o nível de significância de $\alpha=0,05$ e foram realizados nos softwares SPSS (Statistical Package for Social Science) for Windows $^{\circledR}$, versão 18.0 e EQS 6.1 for Windows ${ }^{\circledR}$.

\section{Resultados}

\section{Concentração Populacional das Cidades dos Jogadores}

Os resultados mostraram que $34,0 \%$ dos jogadores nasceram em cidades com até 100 mil habitantes, $35,7 \%$ em cidades entre 100 mil e 1 milhão, e $30,3 \%$ em cidades com mais de 1 milhão de habitantes (vide Tabela 1). Apesar desta distribuição quase equitativa entre estas três faixas populacionais, pode-se verificar diferenças significativas entre as frequências de jogadores nos 18 intervalos populacionais $\left[\chi^{2}=297,227 ; p<0,001\right]$ mostrados na Tabela 1 , sendo que oito destes intervalos (I-1, I-2, I-3, I-4, $\mathrm{I}-5$, I-6, I-17 e I-18) representam $72,8 \%$ das cidades de origem dos jogadores participantes do Campeonato Brasileiro Série A de 2010. Do total de jogadores, $55,3 \%$ nasceram em cidades com até 400 mil habitantes e $17,5 \%$ tiveram suas origens em centros urbanos com mais de seis milhões de pessoas.

Ainda na Tabela 1, é possível verificar uma relação inversa entre as chances de ascensão ao alto rendimento e a população das cidades, com exceção dos intervalos I-12 e I-13. Assim, à medida que população das cidades aumenta as chances de ascensão ao alto rendimento vão sendo reduzidas, i.e., jogadores nascidos no primeiro intervalo populacional possuíram uma chance de profissionalização entre 17 ou 345 indivíduos, já os jogadores nascidos no último intervalo populacional tiveram uma chance de profissionalização entre 166.667 ou 183.960 indivíduos. Esta diferença promove aos jogadores nascidos no último intervalo populacional (I-18) uma redução de chances de se profissionalizar de 533 até 9803 vezes em relação aos jogadores nascidos no primeiro intervalo populacional (I-1). 
Tabela 1. Frequência dos jogadores nos 18 intervalos populacionais do estudo e as suas chances de ascensão ao alto rendimento no futebol.

\begin{tabular}{|c|c|c|c|c|c|c|c|c|c|c|}
\hline \multirow{2}{*}{$\begin{array}{l}\text { Inter- } \\
\text { valos }\end{array}$} & \multirow{2}{*}{ População Cidades } & \multicolumn{5}{|c|}{ Frequência } & \multirow{2}{*}{ Percentual } & \multicolumn{2}{|c|}{$\begin{array}{c}\text { Comparação } \\
\text { intra-intervalos }\end{array}$} & \multirow{2}{*}{$\begin{array}{l}\text { Chances de } \\
\text { Ascensão* }\end{array}$} \\
\hline & & Gol & Def & Meia & Atac & Total & & $\chi^{2}$ & $p$ & \\
\hline $\mathrm{I}-1$ & 1445 e 30.000 & 11 & 34 & 25 & 17 & 87 & 13,5 & 13,736 & 0,003 & 17 a 345 \\
\hline $\mathrm{I}-2$ & 30.001 e 50.000 & 4 & 14 & 16 & 11 & 45 & 7,0 & 7,356 & 0,061 & 667 a 1.111 \\
\hline I-3 & 50.001 e 100.000 & 15 & 29 & 28 & 15 & 87 & 13,5 & 8,402 & 0,038 & 575 a 1.149 \\
\hline $\mathrm{I}-4$ & 100.001 e 200.000 & 7 & 20 & 22 & 13 & 62 & 9,6 & 9,097 & 0,028 & 1.613 a 3.226 \\
\hline $1-5$ & 200.001 e 300.000 & 6 & 9 & 15 & 8 & 38 & 5,9 & 4,737 & 0,192 & 5.263 a 7.895 \\
\hline I-6 & 300.001 e 400.000 & 8 & 12 & 13 & 4 & 37 & 5,8 & 5,486 & 0,139 & 8.108 a 10.811 \\
\hline $1-7$ & 400.001 e 500.000 & 0 & 4 & 6 & 4 & 14 & 2,2 & 0,571 & 0,751 & 28.572 a 35.714 \\
\hline I-8 & 500.001 e 600.000 & 3 & 6 & 7 & 3 & 19 & 3,0 & 2,684 & 0,443 & 26.316 a 31.579 \\
\hline $1-9$ & 600.001 e 700.000 & 1 & 3 & 5 & 2 & 11 & 1,7 & 3,182 & 0,364 & 54.546 a 63.636 \\
\hline $\mathrm{I}-10$ & 700.001 e 800.000 & 1 & 5 & 2 & 2 & 10 & 1,6 & 3,600 & 0,308 & 70.000 a 80.000 \\
\hline $\mid-11$ & 800.001 e 900.000 & 0 & 5 & 7 & 3 & 15 & 2,3 & 1,600 & 0,449 & 53.333 a 60.000 \\
\hline $\mathrm{I}-12$ & 900.001 e1.000.000 & 1 & 7 & 11 & 4 & 23 & 3,6 & 9,522 & 0,023 & 39.130 a 43.478 \\
\hline $\mathrm{I}-13$ & 1.000 .001 e 1.500 .000 & 4 & 13 & 6 & 3 & 26 & 4,0 & 9,385 & 0,025 & 38.462 a 57.692 \\
\hline $\mathrm{I}-14$ & 1.500 .001 e 2.000 .000 & 0 & 3 & 8 & 3 & 14 & 2,2 & 3,571 & 0,168 & 107.143 a 142.857 \\
\hline $\mathrm{l}-15$ & 2.000 .001 e 2.500 .000 & 2 & 6 & 2 & 5 & 15 & 2,3 & 3,400 & 0,334 & 133.333 a 166.667 \\
\hline $\mathrm{I}-16$ & 2.500 .001 e 6.000 .000 & 0 & 10 & 11 & 6 & 27 & 4,3 & 1,556 & 0,459 & 92.593 a 222.222 \\
\hline $\mathrm{I}-17$ & 6.000 .001 e 10.000 .000 & 3 & 14 & 28 & 8 & 53 & 8,2 & 26,472 & $<0,001$ & 113.208 a 188.679 \\
\hline \multirow[t]{2}{*}{$\mathrm{l}-18$} & 10.000 .001 e 11.037 .593 & 7 & 15 & 22 & 16 & 60 & 9,3 & 7,600 & 0,055 & 166.667 a 183.960 \\
\hline & Total & 73 & 209 & 234 & 127 & 643 & 100,0 & & & \\
\hline
\end{tabular}

*As chances de ascensão foram calculadas por uma divisão entre o número de jogadores profissionalizados e a população mínima e máxima contida em cada intervalo populacional.

Nas comparações das distribuições dos jogadores em função do estatuto posicional, verificou-se diferenças significativas para todas as posições na comparação inter-intervalos: goleiros $\left[\chi^{2}=20,082 ; \quad p=0,010\right], \quad$ defesas $\quad\left[\chi^{2}=75,354\right.$; $\mathrm{p}<0,001]$, meias $\left[\chi^{2}=139,128 ; \quad \mathrm{p}<0,001\right]$ e atacantes $\left[\chi^{2}=18,598 ; p=0,046\right]$ e seis diferenças significativas intra-intervalos (l-1, I-3, l-4, l-12, l-13 e I-17), conforme mostra a Tabela 1.

Além disto, foi possível verificar que $90 \%$ dos clubes participantes possuem as suas sedes em cidades com mais de 1 milhão de habitantes e que os cinco estados brasileiros que mais contribuíram para a promoção de jogadores ao alto rendimento foram: São Paulo (28,8\%), Rio de Janeiro (14,3\%), Bahia (8,6\%), Minas Gerais $(7,6 \%)$ e Rio Grande do Sul (6,8\%). Adicionalmente, não se verificou nenhuma relação existente entre a concentração populacional das cidades dos jogadores e a classificação final da equipe no Campeonato Brasileiro Série A de 2010.

\section{Índice de Desenvolvimento Humano das Cidades dos Jogadores}

Em relação aos IDH's das cidades de origem dos jogadores, verificou-se diferenças significativas entre os três intervalos de classificação $\quad\left[\chi^{2}(T 1 \times T 2)=470,114 ; \quad p<0,001\right]$, $\left[\chi^{2}(T 1 x T 3)=65,333 ; \quad p<0,001\right] \quad$ e $\left[\chi^{2}(\right.$ T2xT3 $\left.)=278,338 ; \quad p<0,001\right]$. Do total de jogadores avaliados, $73,1 \%$ são oriundos de cidades com IDH superior ao da média verificada neste estudo $(0,73 \pm 0,08)$, sendo que $82,3 \%$ dos jogadores nasceram em cidades com IDH médio $(0,501-0,800), 15,7 \%$ com IDH alto $(>0,801)$ e somente 2,0\% dos jogadores nasceram em cidades com IDH baixo $(<0,500)$. Dentre os estados brasileiros com maior número de jogadores no alto rendimento verificaram-se os seguintes IDH's: São Paulo $(0,78 \pm 0,03)$, Rio de Janeiro $(0,76 \pm 0,05)$, Bahia $(0,63 \pm 0,09)$, Minas Gerais $(0,73 \pm 0,05)$ e Rio Grande do Sul $(0,76 \pm 0,04)$. Já os dados de IDH das cidades sedes dos 20 clubes participantes desta pesquisa, indicam uma média de $0,82 \pm 0,03$, sendo que $90 \%$ destes clubes estão em cidades com IDH acima de 0,80 .

No que refere à classificação final das equipes no Campeonato Brasileiro Série A de 2010, não se verificou diferenças significativas entre as médias de IDH's apresentadas pelas 20 equipes participantes $(p \leq 0,05)$. Entretanto, foram verificadas 19 diferenças significativas nas comparações das distribuições dos IDH's intraequipe (vide Tabela 2). Já nas comparações interequipes, foi encontrada somente uma diferença significativa no intervalo $\mathrm{T} 3(>0,801)$ da equipe $3^{3}$ Colocada para as demais $\left[\chi^{2}=38,167 ; p=0,006\right]$. 
Tabela 2. Valores mínimos, máximos e médios dos Índices de Desenvolvimento Humano das equipes e as suas frequências nos três intervalos de classificação.

\begin{tabular}{|c|c|c|c|c|c|c|c|c|c|}
\hline \multirow{2}{*}{$\begin{array}{l}\text { CLASSIFICAÇÃO } \\
\text { FINAL }\end{array}$} & \multirow[b]{2}{*}{ Min. } & \multirow[b]{2}{*}{ Max. } & \multirow[b]{2}{*}{$X \pm d p$} & \multicolumn{3}{|c|}{ Tercis dos IDH's } & \multirow[b]{2}{*}{ Total } & \multicolumn{2}{|c|}{ Comparação intra-equipe } \\
\hline & & & & $<0,500$ & $\begin{array}{c}\text { entre } 0,501 \\
\text { e } 0,800\end{array}$ & $>0,801$ & & $\chi^{2}$ & $p$ \\
\hline 1L (Campeão) & 0,48 & 0,84 & $0,75 \pm 0.08$ & 1 & 23 & 7 & 31 & 25,032 & $<0,001$ \\
\hline 2L (Vice-campeão) & 0,55 & 0,81 & $0,72 \pm 0.08$ & 0 & 26 & 2 & 28 & 20,571 & $<0,001$ \\
\hline $3 \mathrm{~L}$ (3잉 lugar) & 0,56 & 0,81 & $0,77 \pm 0.05$ & 0 & 16 & 13 & 29 & 0,310 & 0,577 \\
\hline $4 \mathrm{~L}$ (4 lugar) & 0,53 & 0,84 & $0,75 \pm 0.06$ & 0 & 26 & 6 & 32 & 12,500 & $<0,001$ \\
\hline $5 \mathrm{~L}$ (5 lugar) & 0,54 & 0,82 & $0,72 \pm 0.08$ & 0 & 28 & 1 & 29 & 25,138 & $<0,001$ \\
\hline 6L (6o lugar) & 0,50 & 0,82 & $0,72 \pm 0.07$ & 1 & 26 & 2 & 29 & 41,448 & $<0,001$ \\
\hline $7 \mathrm{~L}$ ( $7^{\circ}$ lugar $)$ & 0,57 & 0,82 & $0,76 \pm 0.05$ & 0 & 24 & 4 & 28 & 14,286 & $<0,001$ \\
\hline 8L (8ㅇ lugar) & 0,43 & 0,89 & $0,72 \pm 0.10$ & 3 & 27 & 2 & 32 & 37,563 & $<0,001$ \\
\hline $9 \mathrm{~L}$ (9o lugar) & 0,51 & 0,81 & $0,74 \pm 0.08$ & 0 & 21 & 7 & 28 & 7,000 & 0,008 \\
\hline $10 \mathrm{~L}$ (10 lugar) & 0,48 & 0,82 & $0,73 \pm 0.10$ & 2 & 19 & 7 & 28 & 16,357 & $<0,001$ \\
\hline $11 \mathrm{~L}$ (11ㅇlugar) & 0,57 & 0,84 & $0,76 \pm 0.06$ & 0 & 27 & 8 & 35 & 10,314 & 0,001 \\
\hline $12 \mathrm{~L}$ (12ㅇ lugar) & 0,48 & 0,81 & $0,69 \pm 0.09$ & 1 & 29 & 3 & 33 & 44,364 & $<0,001$ \\
\hline 13L (13ㅇlugar) & 0,61 & 0,82 & $0,74 \pm 0.07$ & 0 & 23 & 5 & 28 & 11,571 & 0,001 \\
\hline 14L (14이 lugar) & 0,54 & 0,82 & $0,76 \pm 0$. & 0 & 24 & 7 & 31 & 9,323 & 0,002 \\
\hline $15 \mathrm{~L}$ (15ㅇ lugar) & 0,49 & 0,82 & $0,72 \pm 0.08$ & 1 & 27 & 1 & 29 & 46,621 & $<0,001$ \\
\hline $16 \mathrm{~L}$ (16ㅇ lugar) & 0,52 & 0,80 & $0,71 \pm 0.08$ & 0 & 29 & 1 & 30 & 26,133 & $<0,001$ \\
\hline 17L (17이 lugar) & 0,41 & 0,81 & $0,68 \pm 0.11$ & 2 & 34 & 4 & 40 & 48,200 & $<0,001$ \\
\hline 18L (18ㅇ lugar) & 0,47 & 0,84 & $0,74 \pm 0.09$ & 1 & 21 & 8 & 30 & 20,600 & $<0,001$ \\
\hline 19L (19o lugar) & 0,52 & 0,89 & $0,72 \pm 0.09$ & 0 & 29 & 3 & 32 & 21,125 & $<0,001$ \\
\hline $20 \mathrm{~L}$ (20 lugar) & 0,51 & 0,82 & $0,71 \pm 0.08$ & 0 & 26 & 5 & 31 & 14,226 & $<0,001$ \\
\hline GERAL & 0,41 & 0,89 & $0,73 \pm 0,08$ & 12 & 505 & 96 & 613 & 680,891 & $<0,001$ \\
\hline
\end{tabular}

\section{Data de Nascimento dos Jogadores}

As diferenças se tornam mais evidentes quando se compara os quatro trimestres de nascimento dos jogadores (vide Tabela 4). Nestes casos, o quarto trimestre $\left(4^{\circ} \mathrm{T}\right)$ apresentou-se diferente dos demais $1^{\circ} \mathrm{T}\left[\chi^{2}=28,876 ; p<0,001\right]$, 2T $\left[\chi^{2}=21,918 ; \quad p<0,001\right]$ e $3 \mathrm{~T} \quad\left[\chi^{2}=7,879\right.$; $\mathrm{p}=0,005]$, possuindo sempre um número menor de jogadores. Adicionalmente, diferenças estatísticas puderam ser também verificadas entre as frequências de atletas oriundos do $1 \cong T$ para o $3^{\circ} T\left[\chi^{2}=6,840 ; p=0,009\right]$.

Nas comparações entre os trimestres de nascimento em função dos estatutos posicionais dos jogadores, verificaram-se sete diferenças significativas, sendo que todas elas se revelaram favoráveis aos jogadores nascidos no 1ำ (vide Tabela 4). Nestas comparações foi possível verificar que os goleiros apresentaram diferenças do $1^{\circ} \mathrm{T}$ para o $3^{\circ} \mathrm{T}$ e $4^{\circ} \mathrm{T}$, os defensores e meio-

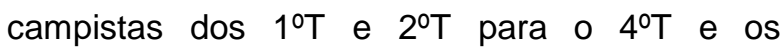
atacantes do $1^{\circ} \mathrm{T}$ para $04^{\circ} \mathrm{T}$.

No que refere à classificação final das equipes no Campeonato Brasileiro Série A de 2010, não se verificou diferenças significativas entre as distribuições das datas de nascimento dos jogadores nas 20 equipes participantes $(p \leq 0,05)$.

Tabela 3. Frequências geral e por estatuto posicional das datas de nascimento dos jogadores pelos quatro trimestres do ano.

\begin{tabular}{|c|c|c|c|c|c|}
\hline Trimestre & Geral & Atacantes & Goleiros & Meio-campistas & Defensores \\
\hline $1^{\circ}$ T (Jan-Mar) & $200(31,1 \%)$ & $42(33,1 \%)$ & $30(41,1 \%)$ & $57(27,3 \%)$ & $71(30,3 \%)$ \\
\hline $2^{\circ} \mathrm{T}$ (Abr-Jun) & $186(28,9 \%)$ & $34(26,8 \%)$ & $17(23,3 \%)$ & $62(29,7 \%)$ & $73(31,2 \%)$ \\
\hline $3^{\circ}$ T (Jul-Set) & $151(23,5 \%)$ & $31(24,4 \%)$ & $14(19,2 \%)$ & $54(25,8 \%)$ & $52(22,2 \%)$ \\
\hline $4^{\circ} \mathrm{T}$ (Out-Dez) & $106(16,5 \%)$ & $20(15,7 \%)$ & $12(16,4 \%)$ & $36(17,2 \%)$ & $38(16,2 \%)$ \\
\hline Total & $643(100,0 \%)$ & $127(100,0 \%)$ & $73(100,0 \%)$ & $234(100,0 \%)$ & $209(100,0 \%)$ \\
\hline
\end{tabular}


Tabela 4. Comparação das frequências geral e por estatuto posicional das datas de nascimento dos jogadores pelos quatro trimestres do ano.

\begin{tabular}{|c|c|c|c|c|c|c|c|c|c|c|}
\hline \multirow{2}{*}{$\begin{array}{c}\text { Comparação entre } \\
\text { Trimestres }\end{array}$} & \multicolumn{2}{|c|}{ Geral } & \multicolumn{2}{|c|}{ Atacantes } & \multicolumn{2}{|c|}{ Goleiros } & \multicolumn{2}{|c|}{ Meio-campistas } & \multicolumn{2}{|c|}{ Defensores } \\
\hline & $x^{2}$ & $p$ & $x^{2}$ & $p$ & $x^{2}$ & $p$ & $x^{2}$ & $p$ & $x^{2}$ & $p$ \\
\hline $1^{\circ} \mathrm{\top} \mathrm{T} \times 2^{\circ} \mathrm{\top} \top$ & 0,508 & 0,476 & 0,842 & 0,359 & 3,596 & 0,058 & 0,028 & 0,868 & 0,210 & 0,647 \\
\hline $1 \stackrel{\circ}{ } \mathrm{T} \times 3^{\circ} \mathrm{T}$ & 6,840 & 0,009 & 1,658 & 0,198 & 5,818 & 0,016 & 2,935 & 0,087 & 0,081 & 0,776 \\
\hline $1 \stackrel{\circ}{\top} \mathrm{T} \times 4^{\circ} \mathrm{T}$ & 28,876 & $<0,001$ & 7,806 & 0,005 & 7,714 & 0,005 & 9,991 & 0,002 & 4,742 & 0,029 \\
\hline $2^{\circ} \mathrm{T} T \times 3^{\circ} \mathrm{T}$ & 3,635 & 0,057 & 0,138 & 0,710 & 0,290 & 0,590 & 3,528 & 0,060 & 0,552 & 0,458 \\
\hline $2^{\circ} \mathrm{\top} T \times 4^{\circ} \mathrm{T}$ & 21,918 & $<0,001$ & 3,630 & 0,057 & 0,862 & 0,353 & 11,036 & 0,001 & 6,898 & 0,009 \\
\hline $3 \stackrel{\circ}{\top} \times 4^{\circ} \div$ & 7,879 & 0,005 & 2,373 & 0,123 & 0,154 & 0,695 & 2,178 & 0,140 & 3,600 & 0,058 \\
\hline
\end{tabular}

\section{Relação entre o Índice de Desenvolvimento Humano, a Concentração Populacional das Cidades e as Datas de Nascimento dos Jogadores}

Os testes de correlação entre estas três variáveis mostraram que a data de nascimento (DN) dos jogadores possui baixa e negativa relação com 0 Índice de Desenvolvimento Humano (IDH) $(r=-0,09 ; \mathrm{ep}=0,04 ; \mathrm{p}=0,03)$ e com a Concentração Populacional das cidades de origem dos jogadores $(r=-0,09$, ep=0,04; $p=0,04)$. Entretanto, foi possível constatar uma correlação alta e positiva para entre o IDH e a Concentração Populacional das cidades de origem dos jogadores $(r=0,69$; $e p=0,02 ; p=0,04)$.

\section{Discussão}

Este trabalho teve por objetivo identificar a possível influência do Índice de Desenvolvimento Humano (IDH) e da data de nascimento (DN) na ascensão de jogadores ao alto nível de rendimento, no contexto do futebol brasileiro. Através dos resultados foi possível verificar que estas duas variáveis exercem influência sobre as possibilidades de jovens jogadores chegarem às principais equipes do futebol brasileiro.

No que refere as condições sócio-econômicas, verificou-se que jogadores advindos de cidades com taxas demográficas abaixo de 200 mil habitantes e com IDH acima de 0,73 reuniram as melhores condições para ascender às equipes profissionais. Esta constatação evidencia que jogadores nascidos nas cidades que possuíam este "perfil", conseguiram aproveitar as "condições sócio-demográficas ideais" durante os primeiros anos do seu estágio de desenvolvimento obtendo consequências positivas para a sua formação esportiva.

As diferentes experiências vivenciadas pelos jogadores nas suas cidades de origem, principalmente, durante a fase de desenvolvimento das capacidades físicas, motoras e cognitivas têm sido reportadas como sendo um fator positivo na formação esportiva de jovens talentos antes dos seus ingressos nos clubes de futebol (KRISTJANSDOTTIR; VILHJALMSSON, 2001; KYTTA, 2002; MALINA et al., 2005; CÔTÉ et al., 2006). Conforme aponta Côté e colaboradores (2006) o espaço físico e o clima psicossocial das grandes e pequenas cidades são diferentes e, consequentemente, as experiências esportivas vivenciadas pelas crianças durante os seus estágios de crescimento são distintas. As vantagens dos grandes centros urbanos residem-se na estruturação das práticas esportivas, através da qualificação das competições, dos programas de desenvolvimento esportivo presentes em escolinhas de esportes e pela supervisão mais qualificada de treinadores/professores (KRISTJANSDOTTIR; VILHJALMSSON, 2001). Por outro lado, as cidades menores propiciam um ambiente mais espaçoso e seguro para as crianças movimentarem-se livremente sem a supervisão de adultos, além de propiciar várias horas de prática da atividade e maior possibilidade de permanência em equipes competitivas da cidade. Estas oportunidades podem ser benéficas para 0 desenvolvimento das habilidades de jogo das crianças, tendo com possível consequência, o aumento da autoconfiança e da motivação para a prática do esporte ao longo do tempo (KYTTA, 2002; STARKES; ERICSSON, 2003; CÔTÉ et al., 2006).

A importância das vivências esportivas informais no processo de formação de jogadores de Futebol tem sido reportada na literatura, principalmente, no que refere ao Futebol de rua (SILVEIRA RAMOS, 2003; FONSECA; GARGANTA, 2006). Este fator tem recebido destaque nas investigações sobre a formação esportiva, devido muitos jogadores de alto nível, entre eles Eusébio, Pelé, Maradona, Cruyff e Zidane, terem declarado que tiveram o início do 
seu processo de formação esportiva nas ruas de suas cidades.

No presente estudo, os resultados indicaram que o processo de formação esportiva dos jogadores do Campeonato Brasileiro Série A de 2010 contemplou tanto o jogo informal quanto a prática deliberada. A evidência de que $69,4 \%$ dos jogadores nasceram em cidades com número de habitantes inferior a $90 \%$ das cidades sedes dos clubes de futebol e que $84,3 \%$ dos jogadores advieram de cidades com valor do IDH abaixo de 0,80, mostra que em algum momento da formação esportiva ocorreu uma migração em massa de jovens talentos das suas cidades de origem para as cidades sedes dos clubes. Estas migrações podem ter ocorrido pelo fato destes clubes de futebol oferecerem melhores condições de treino, no que refere a profissionais qualificados, campos de treino, alojamento, alimentação, entre outras condições que permitiam aos jovens jogadores estarem focados no desenvolvimento das suas habilidades esportivas. A visibilidade social e as possibilidades de fazerem carreira esportiva e serem selecionados, mesmo que nas categorias de base, para jogar na Seleção Brasileira são fatores que também podem ser considerados como motivadores para a migração dos jogadores em idades de formação esportiva.

Os dados deste trabalho mostram ainda que parece existir um valor crítico em relação ao IDH $(<0,41)$ e ao número mínimo de habitantes de uma cidade (<1445 pessoas) para que a mesma possa reunir as mínimas condições necessárias para a formação de um jogador de futebol. De acordo com algumas pesquisas, a falta de infraestrutura destas pequenas cidades pode resultar em baixos investimentos públicos e privados de incentivo à prática esportiva e, consequentemente, poucas oportunidades de vivência prática do esporte e de orientação qualificada (CÔTÉ et al., 2006). Além disto, a escassez de estrutura física e a falta de crianças/amigos para a prática do futebol são fatores que podem inibir outras facilidades encontradas nas pequenas cidades, como a prática por longo período de tempo e o desenvolvimento de habilidades específicas de jogo (URTIS; BIRCH, 1987).

No que concerne a data de nascimento dos jogadores, os resultados deste estudo evidenciaram que este é um fator que pode afetar as oportunidades de acesso ao alto nível de rendimento no Futebol Brasileiro. No geral, os jogadores que nasceram nos primeiros três meses do ano têm mais possibilidades de ascensão às equipes profissionais, do que os seus pares que nasceram nos últimos três meses do mesmo ano. Esta diferença pode ser ainda mais notável nas posições em que o rendimento esportivo está relacionado, principalmente nos primeiros anos de formação, com as qualidades físicas, e.g. goleiro, onde se verifica diferenças entre jogadores nascidos até o mês março com jogadores nascidos após o mês de agosto.

Os resultados deste estudo corroboram as evidências dos efeitos idade relativa (EIR's) em pesquisas realizadas em outros países como Estados Unidos (GLAMSER; VINCENT, 2004; VINCENT; GLAMSER, 2006); Bélgica (HELSEN; WINCKEL; WILLIAMS, 2005), Dinamarca (HELSEN; WINCKEL; WILLIAMS, 2005), Inglaterra (HELSEN; WINCKEL; WILLIAMS, 2005), França (HELSEN; WINCKEL; WILLIAMS, 2005), Alemanha (HELSEN; WINCKEL; WILLIAMS, 2005), Itália (HELSEN; WINCKEL; WILLIAMS, 2005), Holanda (HELSEN; WINCKEL; WILLIAMS, 2005), Portugal (FOLGADO et al., 2006), Espanha (JIMÉNEZ; PAIN, 2008; DIAZ DEL CAMPO et al., 2010), e Suécia (HELSEN; WINCKEL; WILLIAMS, 2005), evidenciando que os EIR's é um fenômeno mundial e que necessita ser tratado e discutido amplamente, de forma a minimizar as suas consequências sobre a formação de jogadores em vários esportes.

No contexto do Futebol Brasileiro, a origem desta disparidade de concentração de jogadores pelos meses do ano pode estar associada ao processo de identificação de jogadores nas categorias de base e aos métodos de avaliação de trabalho dos profissionais (treinadores, preparadores físicos, auxiliares técnicos, entre outros) que lidam diretamente com os jovens jogadores. A pressão exagerada por vitórias e conquistas nas etapas de formação, pode ter reflexos diretos no processo de identificação de talentos que, consequentemente, irá influenciar o processo de formação e seleção do jogador até o mais alto nível de rendimento (DIAZ DEL CAMPO et al., 2010).

Com vista a minimizar a incidência de erros no processo de formação de talentos, alguns dirigentes esportivos têm buscado melhorias nos métodos de avaliação de seus profissionais e nos procedimentos de avaliação dos jogadores. Entretanto, no que concerne aos métodos de 
avaliação dos profissionais, muitos clubes ainda embasam os seus indicadores de avaliação somente nas vitórias em jogos e nos títulos conquistados nas competições de base. Destarte, os métodos de avaliação que consideram apenas estes indicadores orientam-se pela procura de resultados imediatos o que, por sua vez, levam estes profissionais a constituírem e organizarem as equipes em função de uma perspectiva de resultados e não de acordo com os imperativos de formação de jogadores.

A justificativa mais utilizada pelas pessoas que advogam este método de avaliação é que as vitórias fazem parte da formação qualificada dos jovens jogadores e que, por isso, os profissionais devem ser avaliados com base nas vitórias e títulos conquistados nas categorias de base. Entretanto, o que se deve ter em consideração durante 0 processo de formação de jovens talentos é que além das vitórias em jogos e conquistas de campeonatos, existem muitos outros constrangimentos que condicionam a qualificação da formação dos jogadores nas categorias de base, nomeadamente, os que se reportam às valências físico-motoras, táticotécnicas e cognitivas (MALINA et al., 2004; CÔTÉ et al., 2006; GRECO, 2006; GARGANTA, 2009).

Ao assumir que somente os resultados desportivos dos jogos são os indicadores absolutos da qualidade do trabalho desenvolvido nas categorias de base, os clubes aceitam que as comissões técnicas, uma vez pressionadas por resultados imediatos em jogos e competições de base, estejam mais propensas a escolher, orientar e escalar jogadores que possuem um porte físico mais desenvolvido, porque nas fases iniciais de desenvolvimento esta componente se sobrepõe às demais, especialmente se o foco estiver direcionado para as facetas defensivas do jogo (GARGANTA, 2006; COSTA et al., 2010; VAEYENS et al., 2010). Entretanto, Williams (2010) chama a atenção para o fato de que jogadores mais dotados fisicamente nos anos iniciais do processo de desenvolvimento esportivo, especialmente até a categoria de sub15 , serem susceptíveis de não desenvolver toda a capacidade técnica e tática necessária para progredir ao mais alto nível de rendimento do jogo. Tal acontece porque estes jogadores não são defrontados com dificuldades que lhes reclamem aquelas competências no conjunto das suas capacidades para jogar. Em consequência, gera-se nos primeiros anos da formação um "déficit" de desenvolvimento esportivo que, posteriormente, poderá não ser recuperado, ocasionando uma limitação evidente do rendimento esportivo nas categorias de idades mais avançadas.

Outro ponto que também pode contribuir para a ocorrência de falhas no processo de identificação de talentos é a superficialidade dos procedimentos de avaliação de jogadores, principalmente, no primeiro contato dos avaliadores com os jovens jogadores nas chamadas "peneiradas". Atualmente, o jovem talento é comumente avaliado através de alguns parâmetros subjetivos do desempenho esportivo e, principalmente, com base na expressão de qualidades cuja medição e observação são relativamente acessíveis, mas não permite 0 acesso a informação fundamental acerca das competências para jogar. A adoção deste procedimento privilegia, na maioria dos casos, as componentes físicas e técnicas em detrimento das componentes táticas, psicológicas e cognitivas do jogo, assim como dos fatores antropométricos, psicomotores, maturacionais, sociais, econômicos e educacionais que influenciam 0 desenvolvimento esportivo (BLOOM, 1985; MCPHERSON, 1994; REILLY et al., 2000; CÔTÉ; BAKER; ABERNETHY, 2003).

Portanto, esta superficialidade dos procedimentos de avaliação aumenta a probabilidade de ocorrência de erro na identificação do talento e traz consequências ao processo de desenvolvimento esportivo dos jogadores e do esporte. Para os jogadores potencialmente mais talentosos este erro tem um significado negativo e pode ocasionar a falta de oportunidade para a qualificação do seu desenvolvimento esportivo e talvez um abandono precoce do esporte. No caso dos jogadores mais dotados fisicamente no momento da avaliação, este erro beneficia-os, pois permite-lhes a passagem para um contexto mais rico de orientações, competitividade e experiências motoras e esportivas que, por sua vez, lhes irão propiciar o acesso a outras oportunidades de aprimoramento das habilidades específicas do jogo, nomeadamente, estar presente e participar em competições internacionais e seleções que, consequentemente, levarão um mais apurado desenvolvimento esportivo.

Assim, em função da superficialidade dos procedimentos de identificação de talentos e das oportunidades de desenvolvimento concedidas 
aos jogadores mais bem dotados fisicamente, aqueles jogadores mais talentosos no primeiro momento da avaliação que poderiam ser identificados nos procedimentos de avaliação, mas não foram porque não tinham os seus processos de maturação física no mesmo nível dos demais, podem não ter mais chances de participar de treinamentos qualificados e continuar o seu processo de desenvolvimento, porque no momento em que igualarem as valências físicas aos demais jogadores, o que irá fazer a diferença será as experiências vividas até o momento. Neste caso, as oportunidades oferecidas durante o processo irão gerar uma "vantagem cumulativa" aos jogadores escolhidos. Deste modo, a pequena diferença inicial conduziu a oportunidades que aprofundaram ainda mais esta diferença, e esta oportunidade por sua vez conduziu a outras experiências que tornaram a pequena diferença inicial ainda maior, que em pouco tempo transformou o jogador escolhido em um fora-de-série; mas que, todavia, não começou como tal, só foi assim, porque em princípio tinha algo que o destacava um pouco dos demais.

Assim, as cobranças aos profissionais das comissões técnicas somente pelos parâmetros de desempenho em jogo e as deficiências na constituição e aplicação dos procedimentos de avaliação fazem com que jogadores talentosos que poderiam ser melhor desenvolvidos, não recebam esta oportunidade e não tenham oportunidade de continuar o seu desenvolvimento esportivo.

Ao ter em mente que o principal objetivo das categorias de base é formar jogadores, torna-se importante aprimorar os procedimentos de identificação de talentos e aperfeiçoar os métodos de avaliação jogadores e profissionais, de forma que se possa dedicar uma atenção especial à influência da data de nascimento e das condições sócio-econômicas sobre o rendimento esportivo dos jogadores, especialmente nas primeiras fases de formação.

Algumas soluções propostas pela literatura com vistas a reduzir ou extinguir a influência dos efeitos idade relativa passam por alterar a datacorte nas competições e nas equipes de base (HURLEY; LIOR; TRACZE, 2001), subdividir categorias em grupos menores (GLAMSER; VINCENT, 2004), agrupar os jogadores pelos seus rendimentos esportivos (KAISERMAN, 2005) e permitir que jogadores permaneçam em suas categorias ao final do ano cronológico, se o seu rendimento não for compatível com o rendimento da categoria superior (GARCIA; SALVADORES, 2005), ou até mesmo permitir que jogadores avancem mais de uma categoria em correspondência ao seu ano cronológico, se o seu rendimento for maximizado através de experiências com jogadores mais velhos. Além destas, uma sugestão menos onerosa e mais segura para os clubes parece passar pela ampliação das escolinhas de esporte e de parcerias com clubes de várias cidades, o que permitiria ao jovem jogador o acesso a prática esportiva de qualidade, com orientação de profissionais qualificados e aumento da competitividade local. Outra vantagem também estaria associada a possibilidade de manter 0 jovem talento próximo ao seio familiar pelo máximo de tempo possível, o que promoveria a convivência em um ambiente psicossocial mais favorável ao desenvolvimento da sua autoconfiança e motivação para o esporte (CÔTÉ et al., 2006). No que concerne aos clubes, as vantagens seriam poder acompanhar o desenvolvimento deste jogador por um longo período de tempo e poder realizar todos os procedimentos de avaliação necessários para se ter certeza que um jovem talento seria aproveitado em sua plenitude. Com esta medida, o clube estaria assegurando que a formação do jogador seria de qualidade e que jovens talentos seriam dificilmente perdidos.

Outra importante sugestão seria os clubes obterem em seus Departamentos de Prospecção de Talentos instrumentos que pudessem ser aplicados nos momentos de realização das "peneiradas" e na sequência do processo de formação esportiva, permitindo uma avaliação mais completa e criteriosa do jogador no que refere às componentes físicas, técnicas, táticas, psicológicas e cognitivas do jogo e alguns fatores antropométricos, psicomotores, maturacionais, sociais, econômicos e educacionais dos jogadores e que são essenciais no processo de desenvolvimento esportivo. Além disto, a criação de padrões e escalas de referências baseadas nos próprios jogadores do clube seria uma importante iniciativa, de forma que os resultados das avaliações dos jovens talentos possam ser comparados com os perfis de jogadores definidos e preferidos pela direção do clube.

Em relação aos métodos de avaliação da qualidade do trabalho das comissões técnicas, admitimos que os clubes teriam vantagem em 
adotar uma avaliação que tomasse em conta os contributos de formação nas categorias mais jovens, na transição para as categorias de idades mais avançadas, considerando como importante indicador a quantidade e qualidade de jogadores que lograram alcançar as categorias de nível mais elevado.

\section{Conclusões}

A partir dos resultados deste estudo afigura-se plausível concluir que o local e a data de nascimento dos jogadores parecem condicionar o seu sucesso esportivo. Jogadores nascidos nos primeiros meses do ano e em cidades com até 200 mil habitantes e Índice de Desenvolvimento Humano acima de 0,73 parecem reunir condições mais favoráveis para ascender ao alto nível de rendimento no Futebol Brasileiro.

Apesar de terem sido verificadas algumas diferenças intra-equipes, nomeadamente, entre as frequências de data de nascimento dos jogadores e entre as médias do Índice de Desenvolvimento Humano das respectivas cidades, no geral não são notórios os impactos significativos no rendimento das equipes no Campeonato Brasileiro Série A de 2010. A presente constatação revela a importância dos dirigentes e dos profissionais do futebol reforçarem a sua atenção e controle sobre a influência destas duas variáveis na formação dos jovens jogadores. Atendendo que o diagnóstico no alto rendimento fica comprometido em função do nivelamento das equipes, recomenda-se que o mesmo seja efetuado nas categorias de base, de modo a propiciar a promoção de maior número de jogadores talentosos à principal competição do Futebol Brasileiro.

\section{Referências}

ASHER, J.; DAPONTE, B. O. A Hypothetical Cohort Model of Human Development. Human Development Research Paper, ONU, v.40, p.153. 2010.

ASHWORTH, J.; HEYNDELS, B. Selection Bias and Peer Effects in Team Sports: The Effect of Age Grouping on Earnings of German Soccer Players. Journal of Sports Economics, Chicago, v.8, n.4, p.355-377. 2007. http://dx.doi.org/10.1177/1527002506287695

BAKER, J.; CÔTÉ, J.; ABERNETHY, B. SportSpecific practice and the development of expert decision-making in team ball sports. Journal of Applied Sport Psychology, Philadelphia, v.15, p.12-25. 2003.

http://dx.doi.org/10.1080/10413200390180035

BARNSLEY, R. H.; THOMPSON, A. H.; BARNSLEY, P. E. Hockey success and birthdate: The relative age effect. Journal of the Canadian Association of Health, Physical Education and Recreation, Ottawa, v.51, p.23-28. 1985. http://dx.doi.org/10.1177/1012690210371560

\section{BLOOM, B. S. Developing talent in young} people. New York: Ballantine. 1985.

COBLEY, S.; ABRAHAM, C.; BAKER, J. Relative age effects on physical education attainment and school sport representation. Physical Education \& Sport Pedagogy, Worcester v.13, n.3, p.267276. 2008.

http://dx.doi.org/10.1080/17408980701345626

COSTA, I.; GARGANTA, J.; GRECO, P.; MESQUITA, I.; SEABRA, A. Influence of Relative Age Effects and Quality of Tactical Behaviour in the Performance of Youth Soccer Players.

International Journal of Performance Analysis in Sport, Cardiff, v.10, n.2, p.82-97. 2010.

CÔTÉ, J.; BAKER, J.; ABERNETHY, B. From play to practice: A developmental framework for the acquisition of expertise in team sports. In: STARKES J.; ERICSSON, K. A. (Ed.). Expert Performance in Sports: Advances in Research on Sport Expertise. Champaign, IL: Human Kinetics Publishers, 2003, p.89-113.

CÔTÉ, J.; MACDONALD, D. J.; BAKER, J.; ABERNETHY, B. When "where"; is more important than "when": Birthplace and birthdate effects on the achievement of sporting expertise. Journal of Sports Sciences, Londres, v.24, n.10, p.1065-1073. 2006. http://dx.doi.org/10.1080/02640410500432490

CURTIS, J. E.; BIRCH, J. S. Size of community of origin and recruitment to professional and Olympic hockey in North America. Sociology of Sport Journal, Austin, v.4, p.229-244. 1987.

DIAZ DEL CAMPO, D. G.; PASTOR VICEDO, J. C.; GONZALEZ VILLORA, S.; CONTRERAS JORDAN, O. R. The relative age effect in youth soccer players from Spain. Journal of Sports Science \& Medicine, Bursa, v.9, n.2, p.190-198. 2010.

FOLGADO, H. A.; CAIXINHA, P. F.; SAMPAIO, J.; MAÇÃS, V. Efeito da idade cronológica na distribuição dos futebolistas por escalões de formação e pelas diferentes posições especificas. Revista Portuguesa de Ciências do Desporto, Porto v.6, n.3, p.349-355. 2006. 
FONSECA, H.; GARGANTA, J. Futebol de Rua: um Beco com Saída. Lisboa: Visão e Contextos. 2006.

GARCIA, V.; SALVADORES, J. El efecto relativo de la edad en el fútbol. Training Fútbol, v.115, n.36-42. 2005.

GARGANTA, J. Idéias e competências para "pilotar" o jogo de futebol. In: TANI, G.; BENTO, J.O.; PETERSEN, R. (Ed.). Pedagogia do Desporto. Rio de Janeiro: Guanabara Koogan, 2006, p.313-326.

GARGANTA, J. New trends of tactical performance analysis in team sports: bridging the gap between research, training and competition. Revista Portuguesa de Ciências do Desporto, Porto, v.9, n.1, p.81-89. 2009.

GLAMSER, F. D.; VINCENT, J. The relative age effect among elite american youth soccer players. Journal of Sport Behavior, Alabama, v.27, n.1, p.31-38. 2004.

GRECO, P. J. Conhecimento tático-técnico: eixo pendular da ação tática (criativa) nos jogos esportivos coletivos. Revista Brasileira de Educação Física e Esporte, São Paulo, v.20, p.210-212. 2006.

HELSEN, W. F.; STARKES, J. L.; VAN WINCKEL, J. Effect of a change in selection year on success in male soccer players. American Journal of Human Biology, Nova York, v.12, n.6, Nov-Dec, p.729-735. 2000.

HELSEN, W. F.; WINCKEL, J. V.; WILLIAMS, A. $M$. The relative age effect in youth soccer across Europe. Journal of Sports Sciences, Londres, v.23, n.6, p.629-636. 2005.

HURLEY, W.; LIOR, D.; TRACZE, S. A proposal to reduce the age discrimination in Canadian minor hockey. Canadian Public Policy, Montreal, v.27, n.1, p.65-75. 2001.

JIMÉNEZ, I. P.; PAIN, M. T. G. Relative age effect in Spanish association football: Its extent and implications for wasted potential. Journal of Sports Sciences, Londres, v.26, n.10, p.9951003. 2008.

JOHNSON, A.; DOHERTY, P.; FREEMONT, A. Chronological versus skeletal bone age in schoolboy footballers. In: REILLY, T.; KORKUSUZ, F. (Ed.). Science and Football VI Proceedings on the Sixth World Congress of Science and Football. London: Routledge, 2009, p.132-137.
KAISERMAN, K. Skill based division of talent in recreational youth leagues. 2005. Disponível em: $<$ http://goarticles.com/article/Skill-Based-Divisionof-Talent-in-Recreational-Youth-

Leagues/5209419/>. Acesso em: 02 de Maio 2011

KRISTJANSDOTTIR, G.; VILHJALMSSON, R. Sociodemographic differences in patterns of sedentary and physically active behaviour in older children and adolescents. Acta Paediatrica, Bruxelas, v.90, p.429-435. 2001.

KYTTA, M. Affordances of children's environments in the context of cities, small towns, suburbs, and rural villages in Finland and Belarus. Journal of Environmental Psychology, Londres, v.22, p.109-123. 2002. http://dx.doi.org/10.1006/jevp.2001.0249.

MALINA, R.; BOUCHARD, C.; BAR-OR, O. Growth, Maturation and Physical Activity. Champaign, IL: Human Kinectics. 2004.

MALINA, R.; EISENMANN, J. C.; CUMMING, S. P.; RIBEIRO, B.; AROSO, J. Maturity-associated variation in the growth and functional capacities of youth football (soccer) players 13-15 years.

European Journal Applied Physiology, Berlin, v.91, p.555-562. 2004.

MALINA, R. M. Physical growth and biological maturation of young athletes. Exercise and Sport Sciences Reviews, Philadelphia v.22, p.389-434. 1994.

MALINA, R. M.; CUMMING, S. P.; KONTOS, A. P.; EISENMANN, J. C.; RIBEIRO, B.; AROSO, J. Maturity-associated variation in sport-specific skills of youth soccer players aged 13 - 15 years. Journal of Sports Sciences, Londres, v.23, n.5, p.515-522. 2005.

MCPHERSON, S. The Development of Sport Expertise: Mapping the Tactical Domain. Quest, v.46, n.2, p.223-240. 1994. http://dx.doi.org/10.1080/00336297.1994.1048412 $\underline{3}$

MUJIKA, I.; VAEYENS, R.; MATTHYS, S.; SANTISTEBAN, J.; GOIRIENA, J.; PHILIPPAERTS, $R$. The relative age effect in a professional football club setting. Journal of Sports Sciences, Londres, v.27, n.11, p.11531158. 2009.

MUSCH, J.; GRONDIN, S. Unequal competition as an impediment to personal development: A review of the relative age effect in sport.

Developmental Review, Nova York, v.21, n.2, p.147-167. 2001. http://dx.doi.org/10.1006/drev.2000.0516. 
MUSCH, J.; HAY, R. The relative age effect in soccer: cross-cultural evidence for a systematic discrimination against children born late in the competition year. Sociology of Sport Journal, v.16, n.1, p.54-64. 1999.

REILLY, A. T.; WILLIAMS, A. M.; NEVILL, A.; FRANKS, A. A multidisciplinary approach to talent identification in soccer. Journal of Sports

Sciences, Londres, v.18, p.695-702. 2000.

SHEARER, E. The effect of date of birth in teachers' assessments of children. Educational Research, Washington, v.10, p.51-56. 1967.

\section{SILVEIRA RAMOS, F. Futebol da "Rua" à} Competição. Lisboa: Instituto do Desporto de Portugal. 2003.

SOBERLAK, P.; CÔTÉ, J. The developmental activities of elite ice hockey players. Journal of Applied Sport Psychology, Indianapolis v.15, n.1, Mar, p.41-49. 2003.

http://dx.doi.org/10.1080/10413200305401

STARKES, J.; ERICSSON, K. A., Eds. Expert Performance in Sports: Advances in Research on Sport Expertise. Champaign, IL: Human Kinetics Publishersed. 2003.

VAEYENS, R.; LENOIR, M.; WILLIAMS, A. M.; MATTHYS, S.; PHILIPPAERTS, R. M. The mechanisms underpinning decision-making in youth soccer players: an analysis of verbal reports. In: DRUST, B.; REILLY, T.; WILLIAMS, M. (Ed.). International Research in Science and Soccer: Proceedings of the First World Conference on Science and Soccer. London: Routledge, 2010, p.21-28.

VAEYENS, R.; PHILIPPAERTS, R. M.; MALINA, $R$. M. The relative age effect in soccer: A matchrelated perspective. Journal of Sports Sciences, Londres, v.23, n.7, p.747-756. 2005.

VINCENT, J.; GLAMSER, F. D. Gender differences in the relative age effect among US olympic development program youth soccer players. Journal of Sports Sciences, Londres, v.24, n.4, p.405-413. 2006.

WILLIAMS, A. M.; ERICSSON, K. A. Perceptualcognitive expertise in sport: some considerations when applying the expert performance approach. Human Movement Science, Amsterdam, v.24, p.283-307. 2005.

WILLIAMS, J. H. Relative age effect in youth soccer: analysis of the FIFA U17 World Cup competition. Scandinavian Journal of Medicine \& Science in Sports, Copenhagen, v.20, p.502508. 2010.
WILLIAMS, P. H.; DAVIES, P.; EVANS, R.; FERGUSON, N. Season of birth and cognitive development. Nature, Londres v.228, n.5276, p.1033-1036. 1970.

Endereço:

Israel Teoldo da Costa

UFV - Departamento de Educação Física Núcleo de Pesquisa e Estudos em Futebol Av: PH Rolfs, s/n - Campus Universitário Viçosa MG Brasil 36570-000

Telefone: (31)-3899-4394

Fax: (31) 3899-2249

e-mail: israel.teoldo@ufv.br

Recebido em: 9 de junho de 2011.

Aceito em: 11 de dezembro de 2012.

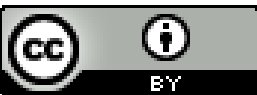

Motriz. Revista de Educação Física. UNESP, Rio Claro, SP, Brasil - elSSN: 1980-6574 - está licenciada sob Creative Commons - Atribuicão 3.0 